\title{
On a non flat Riemannian warped product manifold with respect to quarter-symmetric connection
}

\section{Buddhadev Pal}

Department of Mathematics, Institute of Science, Banaras Hindu University, India email: pal.buddha@gmail.com

\section{Santu Dey}

Department of Mathematics, Bidhan Chandra College, Asansol- 713303, India santu.mathju@gmail.com

\author{
Sampa Pahan \\ Department of Mathematics, \\ Mrinalini Datta Mahavidyapith, \\ Birati, Kolkata-700051, India \\ email: sampapahan25@gmail.com
}

\begin{abstract}
In this paper, we study generalized quasi-Einstein warped products with respect to quarter symmetric connection for dimension $n \geq$ 3 and Ricci-symmetric generalized quasi-Einstein manifold with quarter symmetric connection. We also investigate that in what conditions the generalized quasi-Einstein manifold to be nearly Einstein manifold with respect to quarter symmetric connection. Example of warped product on generalized quasi-Einstein manifold with respect to quarter symmetric connection are also discussed.
\end{abstract}

\section{Introduction}

A Riemannian manifold $\left(M^{n}, g\right),(n>2)$ is Einstein if its Ricci tensor $S$ of type $(0,2)$ is of the form $S=\alpha g$, where $\alpha$ is smooth function, which turns

2010 Mathematics Subject Classification: 53C25, 53B30, 53C15

Key words and phrases: Einstein manifold, quasi-Einstein manifold, nearly quasi-Einstein manifold, generalized quasi-Einstein manifold, warped product manifold 
into $S=\frac{r}{n} g, r$ being the scalar curvature of the manifold. The notion of quasi Einstein manifold was introduced by M. C. Chaki and R. K. Maity [2]. A nonflat Riemannian manifold $\left(M^{n}, g\right),(n>2)$ is defined to be a quasi Einstein manifold if its Ricci tensor $S$ of type $(0,2)$ is not identically zero and satisfies the condition

$$
S(X, Y)=\alpha g(X, Y)+\beta A_{1}(X) A_{1}(Y)
$$

where $\alpha, \beta$ are scalars of which $\beta \neq 0$ and $A_{1}$ is a non-zero 1-form such that $g(X, U)=A_{1}(X)$ for all vector fields $X$ with $g(U, U)=1$. Such an $n$ dimensional quasi-Einstein manifold is denoted by $(\mathrm{QE})_{n}$.

In [5], De and Ghosh introduced generalized quasi-Einstein manifold, denoted by $\mathrm{G}(\mathrm{QE})_{\mathfrak{n}}$, where the Ricci tensor $S$ of type $(0,2)$ which is not identically zero satisfies the condition

$$
S(X, Y)=\alpha g(X, Y)+\beta A_{1}(X) A_{1}(Y)+\gamma B_{1}(X) B_{1}(Y)
$$

where $\alpha, \beta, \gamma$ are scalars such that $\beta, \gamma$ are nonzero and $A_{1}, B_{1}$ are two nonzero 1 -forms such that

$$
g(X, \mu)=A_{1}(X) \quad \text { and } \quad g(X, \rho)=B_{1}(X),
$$

$\mu, \rho$ being unit vectors which are orthogonal, i.e., $g(\mu, \rho)=0$.

Here $\alpha, \beta, \gamma$ are called the associated scalars, and $A_{1}, B_{1}$ are called the associated main and auxiliary 1 -forms respectively, $\mu, \rho$ are called the main and the auxiliary generators of the manifold.

The notion of warped product generalizes that of a surface of revolution. It was introduced in [1] for studying manifolds of negative curvature. Let $\left(\mathrm{B}, \mathrm{g}_{\mathrm{B}}\right)$ and $\left(\mathrm{F}, \mathrm{g}_{\mathrm{F}}\right)$ be two Riemannian manifolds and $\mathrm{f}$ is a positive differentiable function on $B$. Consider the product manifold $B \times F$ with its projections $\pi: \mathrm{B} \times \mathrm{F} \rightarrow \mathrm{B}$ and $\sigma: \mathrm{B} \times \mathrm{F} \rightarrow \mathrm{F}$. The warped product $\mathrm{B} \times{ }_{f} \mathrm{~F}$ is the manifold $\mathrm{B} \times \mathrm{F}$ with the Riemannian structure such that $\|X\|^{2}=\left\|\pi^{*}(X)\right\|^{2}+f^{2}(\pi(p))\left\|\sigma^{*}(X)\right\|^{2}$, for any vector field $X$ on $M$. Thus we have

$$
g=g_{B}+f^{2} g_{F}
$$

holds on $M$. The function $f$ is called the warping function of the warped product [9].

Since $B \times_{f} F$ is a warped product, then we have $\nabla_{X} Z=\nabla_{Z} X=(X \ln f) Z$ for unit vector fields $X, Z$ on $B$ and $F$, respectively. Hence, we find $K(X \wedge Z)=$ $g\left(\nabla_{Z} \nabla_{X} X-\nabla_{X} \nabla_{Z} X, Z\right)=(1 / f)\left\{\left(\nabla_{X} X_{f}-X^{2} f\right\}\right.$. If we chose a local orthonormal 
frame $e_{1}, \ldots, e_{n}$ such that $e_{1}, \ldots, e_{n_{1}}$ are tangent to $B$ and $e_{n_{1}+1}, \ldots, e_{n}$ are tangent to $F$, then we have

$$
\frac{\Delta f}{f}=\sum_{i=1}^{n} K\left(e_{i} \wedge e_{j}\right),
$$

for each $s=n_{1}+1, \ldots, n[9]$.

In 1924, Friedmann and Schouten introduced the notion of a semi-symmetric linear connection on a differentiable manifold [15]. In 1975, Golab introduced the definition of a quarter-symmetric linear connection on a differentiable manifold which is a generalization of semi-symmetric connection in [8]. Many authors like Q. Qu and Y. Wang [14], S. Pahan et al. [16, 17] and S. Dey et al. [18] studied on warped product manifolds with affine connections.

In this paper we study generalized quasi-Einstein warped products with respect to quarter symmetric connection. We discuss some preliminary concepts and results which are useful for proving our main results. We obtain a necessary and sufficient condition for the warped product manifold to be a generalized quasi-Einstein manifold with respect to a quarter-symmetric connection. Next we prove generalized quasi-Einstein manifold with respect to quarter symmetric connection to be nearly quasi Einstein manifold with respect to quarter symmetric connection under some certain conditions. In the last section we give an example of warped product on generalized quasi-Einstein manifold with respect to quarter symmetric connection.

\section{Preliminaries}

Let $\left(M^{n}, g\right)$ be a Riemannian manifold with the Levi-Civita connection $\nabla$. A linear connection $\breve{\nabla}$ on $\left(M^{n}, g\right)$ is said to be a quarter-symmetric connection if its torsion tensor $\mathrm{T}$ with respect to the connection $\breve{\nabla}$ defined by

$$
\mathrm{T}(\mathrm{X}, \mathrm{Y})=\breve{\nabla}_{X} \mathrm{Y}-\breve{\nabla}_{Y} \mathrm{X}-[\mathrm{X}, \mathrm{Y}]
$$

satisfies

$$
\mathrm{T}(\mathrm{X}, \mathrm{Y})=\omega(\mathrm{Y}) \phi X-\omega(\mathrm{X}) \phi \mathrm{Y}
$$

where $\omega$ is a 1-form on $M^{n}$ with the associated vector field $P$ defined by $\omega(X)=g(X, P)$, for all vector field $X$, and $\phi$ is a $(1,1)$ tensor field.

A quarter-symmetric connection $\breve{\nabla}$ is called a quarter-symmetric metric connection if $\breve{\nabla} \mathrm{g}=0 . \breve{\nabla}$ is called a quarter-symmetric non-metric connection 
if $\breve{\nabla} \mathrm{g} \neq 0$. The relation between a quarter-symmetric connection $\breve{\nabla}$ and the Levi-Civita connection $\nabla$ of $M^{n}$ is given by [19]

$$
\breve{\nabla} \mathrm{X} Y=\nabla_{\mathrm{X}} \mathrm{Y}+\lambda_{1} \omega(\mathrm{Y}) \mathrm{X}-\lambda_{2} \mathrm{~g}(\mathrm{X}, \mathrm{Y}) \mathrm{P},
$$

where $g(X, P)=\omega(X)$ and $\lambda_{1} \neq 0, \lambda_{2} \neq 0$ are scalar functions.

We can easily see that: when $\lambda_{1}=\lambda_{2}=1, \breve{\nabla}$ is a semi-symmetric metric connection.

When $\lambda_{1}=\lambda_{2} \neq 1, \breve{\nabla}$ is a quarter-symmetric metric connection.

When $\lambda_{1} \neq \lambda_{2}, \breve{\nabla}$ is a quarter-symmetric non-metric connection.

Further, a relation between the curvature tensors $R$ and $\breve{R}$ of type $(1,3)$ of the connections $\nabla$ and $\breve{\nabla}$ respectively is given by [19],

$$
\begin{aligned}
\breve{R}(X, Y) Z= & R(X, Y) Z+\lambda_{1} g\left(Z, \nabla_{X} P\right) Y-\lambda_{2} g\left(Z, \nabla_{Y} P\right) X+\lambda_{2}\left[g(X, Z) \nabla_{Y} P\right. \\
& \left.-g(Y, Z) \nabla_{X} P\right]+\lambda_{1} \lambda_{2} \omega(P)[g(X, Z) Y-g(Y, Z) X] \\
& +\lambda_{2}^{2}[g(Y, Z) \omega(X)-g(X, Z) \omega(Y)] P+\lambda_{1}^{2} \omega(Z)[\omega(Y) X \\
& -\omega(X) Y]
\end{aligned}
$$

for vector fields $X, Y, Z$ on $M$.

\section{Generalized quasi-Einstein manifold with respect to quarter-symmetric connection}

In this section, we consider the following propositions from Proposition 3.5., Proposition 3.6., Proposition 3.7., Proposition 3.8. of [14], which will be helpful to prove our main results. Here we consider generalized quasi-Einstein warped product manifolds and prove some results concerning these type manifolds.

Theorem 1 Let $(\mathrm{M}, \mathrm{g})$ be a warped product $\mathrm{I} \times_{\mathrm{f}} \mathrm{F}$ where $\mathrm{I}$ is an open interval in $\mathbb{R}, \operatorname{dim} \mathrm{I}=1$ and $\operatorname{dim} \mathrm{F}=\overline{\mathrm{n}}-1, \overline{\mathrm{n}} \geq 3$. Then $(\mathrm{M}, \mathrm{g})$ is a generalized quasi-Einstein manifold with respect to a quarter-symmetric connection if and only if $\mathrm{F}$ is a generalized quasi-Einstein manifold for $\mathrm{P}=\frac{\partial}{\partial \mathrm{t}}$ with respect to the Levi-Civita connection or the warping function $\mathrm{f}$ is a constant on I for $\mathrm{P} \in \chi(\mathrm{F}), \lambda_{2} \neq(\overline{\mathrm{n}}-1) \lambda_{1}$.

Proof. Assume that $P \in \chi(B)$ and taking $f=e^{\frac{q}{2}}$ and using the Proposition 3.1. of [16], we get

$$
\breve{S}\left(\frac{\partial}{\partial t}, \frac{\partial}{\partial t}\right)=(1-\bar{n})\left[\frac{1}{2} q^{\prime \prime}+\frac{1}{4} q^{\prime 2}-\frac{1}{2} \lambda_{2} q^{\prime}+\lambda_{1} \lambda_{2}-\lambda_{1}^{2}\right] g_{I}\left(\frac{\partial}{\partial t}, \frac{\partial}{\partial t}\right),
$$




$$
\begin{gathered}
\breve{S}\left(\frac{\partial}{\partial t}, V\right)=0, \\
\breve{S}(V, W)=S^{F}(V, W)+e^{q}\left[\frac{\bar{n}-1}{4}\left(q^{\prime}\right)^{2}+\frac{1}{2}\left[(\bar{n}-1) \lambda_{1}+(\bar{n}-2) \lambda_{2}\right] q^{\prime}\right. \\
\left.+\lambda_{2}^{2}+\frac{1}{2} q^{\prime \prime}+(1-\bar{n}) \lambda_{1} \lambda_{2}\right] g_{F}(V, W),
\end{gathered}
$$

for vector fields $\mathrm{V}, \mathrm{W}$ on $\mathrm{F}$.

Since $M$ is generalized quasi-Einstein admitting quarter-symmetric connection, from (2) we have

$$
\breve{S_{M}}\left(\frac{\partial}{\partial t}, \frac{\partial}{\partial t}\right)=\alpha g\left(\frac{\partial}{\partial t}, \frac{\partial}{\partial t}\right)+\beta A_{1}\left(\frac{\partial}{\partial t}\right) A_{1}\left(\frac{\partial}{\partial t}\right)+\gamma B_{1}\left(\frac{\partial}{\partial t}\right) B_{1}\left(\frac{\partial}{\partial t}\right),
$$

and

$$
\breve{S_{M}}(V, W)=\alpha g(V, W)+\beta A_{1}(V) A_{1}(W)+\gamma B_{1}(V) B_{1}(W)
$$

Decomposing the vector fields $\mathrm{U}$ and $\mathrm{U}$ uniquely into its components $\mathrm{U}_{\mathrm{I}}, \mathrm{U}_{\mathrm{F}}$ and $\mathrm{U}_{\mathrm{I}}, \mathrm{U}_{\mathrm{F}}$ on $\mathrm{I}$ and $\mathrm{F}$, respectively, we can write $\mathrm{U}=\mathrm{U}_{\mathrm{I}}+\mathrm{U}_{\mathrm{F}}$ and $\mathrm{U}=\mathrm{U}_{\mathrm{I}}+\mathrm{U}_{\mathrm{F}}$ and also $\mathcal{U}^{\prime}=\eta_{2} \frac{\partial}{\partial t}+U_{F}$, where $\eta_{1}$ and $\eta_{2}$ are functions on $M$. Then we can write

$$
\begin{aligned}
& A_{1}\left(\frac{\partial}{\partial t}\right)=g\left(\frac{\partial}{\partial t}, u\right)=\eta_{1}, \\
& B_{1}\left(\frac{\partial}{\partial t}\right)=g\left(\frac{\partial}{\partial t}, u ́\right)=\eta_{2} .
\end{aligned}
$$

On the other hand, by the use of (3) and (12), the equations (10) and (11) reduce to

$$
\breve{S_{M}}\left(\frac{\partial}{\partial t}, \frac{\partial}{\partial t}\right)=\alpha+\beta \eta_{1}^{2}+\gamma \eta_{2}^{2}
$$

and

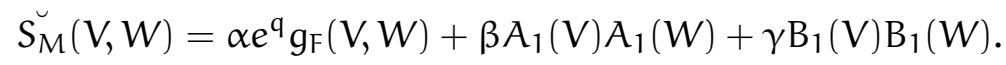

Comparing the right hand side of the equations (7) and (13) we get

$$
\alpha+\beta \eta_{1}^{2}+\gamma \eta_{2}^{2}=-\frac{n-1}{4}\left[2 q^{\prime \prime}+\left(q^{\prime}\right)^{2}\right]
$$

Similarly, comparing the right hand sides of (9) and (14) we obtain

$$
\begin{aligned}
S_{F}(V, W)= & e^{q}\left[\alpha-\left\{\frac{\bar{n}-1}{4}\left(q^{\prime}\right)^{2}+\frac{1}{2}\left\{(\bar{n}-1) \lambda_{1}+(\bar{n}-2) \lambda_{2}\right\} q^{\prime}\right.\right. \\
& \left.\left.+\lambda_{2}^{2}+\frac{1}{2} q^{\prime \prime}+(1-\bar{n}) \lambda_{1} \lambda_{2}\right\}\right] g_{F}(V, W) \\
& +\beta A_{1}(V) A_{1}(W)+\gamma B(V)_{1} B_{1}(W),
\end{aligned}
$$


which gives that $\mathrm{F}$ is a generalized quasi-Einstein manifold with respect to the Levi-Civita connection for $\mathrm{P} \in \chi(\mathrm{B})$.

Taking $\mathrm{P} \in \chi(\mathrm{F})$ and by the use of Proposition 3.1. of [16], we get

$$
\breve{S}\left(\frac{\partial}{\partial t}, V\right)=\frac{q^{\prime}}{2}\left[(\bar{n}-1) \lambda_{1}-\lambda_{2}\right] \omega(V)
$$

and

$$
\breve{S}\left(V, \frac{\partial}{\partial t}\right)=\frac{q^{\prime}}{2}\left[\lambda_{2}-(\bar{n}-1) \lambda_{1}\right] \omega(V),
$$

for any vector field $V \in \chi(F)$.

Since $M$ is a generalized quasi-Einstein manifold, we have

$$
\breve{S}\left(\frac{\partial}{\partial t}, V\right)=\tilde{S}\left(V, \frac{\partial}{\partial t}\right)=\alpha g\left(V, \frac{\partial}{\partial t}\right)+\beta A_{1}(V) A_{1}\left(\frac{\partial}{\partial t}\right)+\gamma B_{1}(V) B_{1}\left(\frac{\partial}{\partial t}\right)
$$

Now $g\left(V, \frac{\partial}{\partial t}\right)=0$ as $\frac{\partial}{\partial t} \in \chi(B)$ and $V \in \chi(F)$.

Hence, from the last equation, we get

$$
\breve{S}\left(\frac{\partial}{\partial t}, V\right)=\breve{S}\left(V, \frac{\partial}{\partial t}\right)=\beta A_{1}(V) A_{1}\left(\frac{\partial}{\partial t}\right)++\gamma B_{1}(V) B_{1}\left(\frac{\partial}{\partial t}\right) .
$$

Therefore, we have

$$
\begin{aligned}
& \beta A_{1}(V) A_{1}\left(\frac{\partial}{\partial t}\right)+\gamma B_{1}(V) B_{1}\left(\frac{\partial}{\partial t}\right)=\frac{q^{\prime}}{2}\left[(\bar{n}-1) \lambda_{1}-\lambda_{2}\right] \omega(V), \\
& \beta A_{1}(V) A_{1}\left(\frac{\partial}{\partial t}\right)+\gamma B_{1}(V) B_{1}\left(\frac{\partial}{\partial t}\right)=\frac{q^{\prime}}{2}\left[\lambda_{2}-(\bar{n}-1) \lambda_{1}\right] \omega(V) .
\end{aligned}
$$

From the equations (21) and (22), we get

$$
q^{\prime}=0,
$$

when $\lambda_{2}-(\bar{n}-1) \lambda_{1} \neq 0$. It follows that $q$ is a constant on $I$. Then $f$ is constant on I. This completes the proof.

Now, we consider the warped product $M=B \times_{f} I$ with $\operatorname{dim} B=\bar{n}-1$, $\operatorname{dim} I=1, \bar{n} \geq 3$. Under this assumption, we obtain the following theorem.

Theorem 2 Let $(\mathrm{M}, \mathrm{g})$ be a warped product $\mathrm{B} \times_{\mathrm{f}} \mathrm{I}$, where $\operatorname{dim} \mathrm{I}=1$ and $\operatorname{dim} \mathrm{B}=\mathrm{n}-1, \mathrm{n} \geq 3$, then 
i) if $(\mathrm{M}, \mathrm{g})$ is a generalized quasi-Einstein manifold with respect to a quartersymmetric connection, $\mathrm{P} \in \chi(\mathrm{B})$ is parallel on $\mathrm{B}$ with respect to the LeviCivita connection on $\mathrm{B}$ and $\mathrm{f}$ is a constant on $\mathrm{B}$, then,

$$
\left.\alpha=\left[(n-1) \lambda_{1} \lambda_{2}-\lambda_{2}^{2}\right)\right] \omega(P) .
$$

ii) If $(\mathrm{M}, \mathrm{g})$ is a generalized quasi-Einstein manifold with respect to a quartersymmetric connection for $\mathrm{P} \in \chi(\mathrm{I})$, and $\lambda_{2} \neq(\mathrm{n}-1) \lambda_{1}$ then $\mathrm{f}$ is a constant on $\mathrm{B}$.

iii) If $\mathrm{f}$ is a constant on $\mathrm{B}$ and $\mathrm{B}$ is a generalized quasi-Einstein manifold with respect to the Levi-Civita connection for $\mathrm{P} \in \chi(\mathrm{I})$, then $\mathrm{M}$ is a generalized quasi-Einstein manifold with respect to a quarter-symmetric connection.

Proof. Assume that $(M, g)$ is a generalized quasi-Einstein manifold with respect to a quarter-symmetric connection. Then we write

$$
\breve{S}(X, Y)=\alpha g(X, Y)+\beta A_{1}(X)_{1} A(Y)+\gamma B_{1}(X) B_{1}(Y) .
$$

Decomposing the vector field $\mathrm{U}$ and $\mathrm{V}$ uniquely into its components $\mathrm{U}_{\mathrm{B}}$ and $\mathrm{U}_{\mathrm{I}}$ on $\mathrm{B}$ and $\mathrm{I}$, respectively, we have

$$
\mathrm{U}=\mathrm{U}_{\mathrm{B}}+\mathrm{U}_{\mathrm{I}}, \quad \mathrm{V}=\mathrm{V}_{\mathrm{B}}+\mathrm{V}_{\mathrm{I}}
$$

Since $\operatorname{dim} I=1$, we can take $U=U_{B}+\eta_{1} \frac{\partial}{\partial t}$ and $V=V_{B}+\eta_{2} \frac{\partial}{\partial t}$, where $\eta_{1}, \eta_{2}$ is a functions on M. From (23), (24) and Proposition 3.1. of [16], we have

$$
\begin{aligned}
\breve{S}^{B}(X, Y)= & \alpha g_{B}(X, Y)+\beta g_{B}\left(X, U_{B}\right) g_{B}\left(Y, U_{B}\right)+\gamma g_{B}\left(X, V_{B}\right) g_{B}\left(Y, V_{B}\right) \\
& -\left[\frac{H^{f}(X, Y)}{f}+\lambda_{2} \frac{P f}{f} g(X, Y)+\lambda_{1} \lambda_{2} \omega(P) g(X, Y)\right. \\
& \left.+\lambda_{1} g\left(Y, \nabla_{X} P\right)-\lambda_{1}^{2} \omega(X) \omega(Y)\right] .
\end{aligned}
$$

By contraction over $\mathrm{X}$ and $\mathrm{Y}$, we get

$$
\begin{aligned}
\breve{r}^{B}= & \alpha(n-1)+\beta g_{B}\left(U_{B}, U_{B}\right)+\gamma g_{B}\left(X, V_{B}\right) g_{B}\left(Y, V_{B}\right) \\
& -\left[\frac{\Delta_{B} f}{f}+\lambda_{2}(n-1) \frac{P f}{f}+\left[(n-1) \lambda_{1} \lambda_{2}-\lambda_{1}^{2}\right] \omega(P)+\lambda_{1} \sum_{i=1}^{n-1} g\left(e_{i}, \nabla_{e_{i}} P\right)\right] .
\end{aligned}
$$


Also from (23), we have

$$
\breve{r}^{M}=\alpha n+\beta g_{B}\left(U_{B}, U_{B}\right)+\gamma g_{B}\left(X, V_{B}\right) g_{B}\left(Y, V_{B}\right) .
$$

Now, putting the value of (27) in (26), we get

$$
\begin{aligned}
\breve{r}^{\mathrm{B}}= & \breve{r}^{M}-\alpha-\frac{\Delta_{\mathrm{B}} f}{f}-\lambda_{2}(n-1) \frac{P f}{f}-\left[(n-1) \lambda_{1} \lambda_{2}-\lambda_{1}^{2}\right] \omega(P) \\
& \left.-\lambda_{1} \sum_{i=1}^{n-1} g\left(e_{i}, \nabla_{e_{i}} P\right)\right] .
\end{aligned}
$$

On the other hand, from Proposition 1., we get

$$
\begin{aligned}
\breve{r}^{M}= & \breve{r}^{B}+(n-1)\left(\lambda_{1}+\lambda_{2}\right) \frac{P f}{f} \\
& +2 \frac{\Delta_{B} f}{f}+\left[2(n-1) \lambda_{1} \lambda_{2}-\left(\lambda_{1}^{2}+\lambda_{2}^{2}\right)\right] \omega(P)+\left(\lambda_{1}+\lambda_{2}\right) \sum_{i=1}^{n-1} g\left(\nabla_{e_{i}} P, e_{i}\right) .
\end{aligned}
$$

Then from the above two relations, we get

$$
\begin{aligned}
& \left.\alpha+\frac{\Delta_{B} f}{f}+\lambda_{2}(n-1) \frac{P f}{f}+\left[(n-1) \lambda_{1} \lambda_{2}-\lambda_{1}^{2}\right] \omega(P)+\lambda_{1} \sum_{i=1}^{n-1} g\left(e_{i}, \nabla_{e_{i}} P\right)\right] \\
& =(n-1)\left(\lambda_{1}+\lambda_{2}\right) \frac{P f}{f}+2 \frac{\Delta f}{f}+\left[2(n-1) \lambda_{1} \lambda_{2}-\left(\lambda_{1}^{2}+\lambda_{2}^{2}\right)\right] \omega(P) \\
& \quad+\left(\lambda_{1}+\lambda_{2}\right) \sum_{i=1}^{n-1} g\left(\nabla_{e_{i}} P, e_{i}\right) .
\end{aligned}
$$

Since $\mathrm{P} \in \chi(\mathrm{B})$ is parallel and $\mathrm{f}$ is a constant on $\mathrm{B}$, then we get

$$
\left.\alpha=\left[(n-1) \lambda_{1} \lambda_{2}-\lambda_{2}^{2}\right)\right] \omega(P) .
$$

ii) Let $\mathrm{P} \in \chi(\mathrm{I})$. By the use of Proposition 3.1. of [16], we get

$$
\breve{S}(X, P)=\left[(n-1) \lambda_{1}-\lambda_{2}\right] \omega(P) \frac{X f}{f},
$$

and

$$
\breve{S}(P, X)=\left[\lambda_{2}-(n-1) \lambda_{1}\right] \omega(P) \frac{X f}{f} .
$$


Since $M$ is a generalized quasi-Einstein manifold, we have

$$
\breve{S}(X, P)=\breve{S}(P, X)=\alpha g(P, X)+\beta A_{1}(P) A_{1}(X)+\gamma B_{1}(P) B_{1}(X)
$$

Again, we have $g(P, X)=0$ for $X \in \chi(B)$ and $P \in \chi(I)$. Hence, we have

$$
X f=0,
$$

where $\lambda_{2} \neq(n-1) \lambda_{1}$. This implies that $f$ is a constant on $B$.

iii) Assume that $B$ is a generalized quasi-Einstein manifold with respect to the Levi-Civita connection. Then we have

$$
S^{B}(X, Y)=\alpha g(X, Y)+\beta A_{1}(X) A_{1}(Y)+\gamma B_{1}(X) B_{1}(Y)
$$

for vector fields $X, Y$ tangent to $B$.

From Proposition 3.1. of [16], we get

$$
\breve{S}^{M}(X, Y)=S^{B}(X, Y)+\left[(n-1) \lambda_{1} \lambda_{2}-\lambda_{2}^{2}\right] \omega(P) g(X, Y)+\frac{H^{f}(X, Y)}{f},
$$

for any vector field $P \in \chi(I)$. Since $f$ is a constant, $H^{f}(X, Y)=0$ for all $X, Y \in$ $x(B)$.

The above equation reduces to

$$
\breve{S}^{M}(X, Y)=S^{B}(X, Y)+\left[(n-1) \lambda_{1} \lambda_{2}-\lambda_{2}^{2}\right] \omega(P) g(X, Y) .
$$

Using the value of (31) in (32), we get

$\breve{S}^{M}(X, Y)=\left\{\alpha+\left[(n-1) \lambda_{1} \lambda_{2}-\lambda_{2}^{2}\right] \omega(P)\right\} g(X, Y)+\beta A_{1}(X) A_{1}(Y)+\gamma B_{1}(X) B_{1}(Y)$

which shows that $M$ is a generalized quasi-Einstein manifold with respect to a quarter-symmetric connection.

Next we find the relation between scalars of generalized quasi-Einstein manifold with respect to a quarter-symmetric connection.

Suppose the generator $U$ is a parallel vector field, then $\breve{R}(X, Y) U=0$. Hence

$$
\breve{S}(X, u)=0 .
$$

Let

$$
\mathrm{U}=\mathrm{U}_{\mathrm{B}}+\mathrm{f}^{2} \mathrm{U}_{\mathrm{F}}, \quad \mathrm{V}=\mathrm{V}_{\mathrm{B}}+\mathrm{f}^{2} \mathrm{~V}_{\mathrm{F}}
$$


From (2), we have

$$
\breve{S}_{M}(X, Y)=\alpha g(X, Y)+\beta A_{1}(X) A_{1}(Y)+\gamma B_{1}(X) B_{1}(Y) .
$$

Putting $\mathrm{Y}=\mathrm{U}$ and using (35), we have

$$
\begin{aligned}
\breve{S}_{M}(X, U) & =\alpha g(X, U)+\beta A_{1}(X) A_{1}(U)+\gamma B_{1}(X) B_{1}(U) \\
& =\left\{\alpha+\beta\left(f^{4}+1\right)\right\} g_{F}\left(X, U_{F}\right) f^{2},
\end{aligned}
$$

where $X \in \chi(F)$ and $Y \in \chi(B)$. From (9), we have

$$
\begin{aligned}
\breve{S_{M}}(X, Y)= & S^{F}(X, Y)+e^{q}\left[\frac{n-1}{4}\left(q^{\prime}\right)^{2}+\frac{1}{2}\left\{(n-1) \lambda_{1}+(n-2) \lambda_{2}\right\} q^{\prime}\right. \\
& \left.+\lambda_{2}^{2}+\frac{1}{2} q^{\prime \prime}+(1-n) \lambda_{1} \lambda_{2}\right] g_{F}(X, Y),
\end{aligned}
$$

for vector fields $X, Y$ on $F$.

As $\mathrm{U}$ is parallel to $\mathrm{F}$, we have from (37)

$$
\begin{aligned}
\breve{S_{M}}(X, U)= & e^{q}\left[\frac{n-1}{4}\left(q^{\prime}\right)^{2}+\frac{1}{2}\left\{(n-1) \lambda_{1}+(n-2) \lambda_{2}\right\} q^{\prime}+\lambda_{2}^{2}+\frac{1}{2} q^{\prime \prime}\right. \\
& \left.+(1-n) \lambda_{1} \lambda_{2}\right] g_{F}\left(X, U_{B}+f^{2} U_{F}\right), \\
= & f^{2} e^{q}\left[\frac{n-1}{4}\left(q^{\prime}\right)^{2}+\frac{1}{2}\left\{(n-1) \lambda_{1}+(n-2) \lambda_{2}\right\} q^{\prime}\right. \\
& \left.+\lambda_{2}^{2}+\frac{1}{2} q^{\prime \prime}+(1-n) \lambda_{1} \lambda_{2}\right] g_{F}(X, U)
\end{aligned}
$$

Now comparing (36) and (38), we have

$$
\begin{aligned}
\left\{\alpha+\beta\left(f^{4}+1\right)\right\}= & e^{q}\left[\frac{n-1}{4}\left(q^{\prime}\right)^{2}+\frac{1}{2}\left\{(n-1) \lambda_{1}+(n-2) \lambda_{2}\right\} q^{\prime}\right. \\
& \left.+\lambda_{2}^{2}+\frac{1}{2} q^{\prime \prime}+(1-n) \lambda_{1} \lambda_{2}\right]
\end{aligned}
$$

So, we get the relation between two non-zero smooth functions $a$ and $b$ of the manifold $M$ with respect to a quarter-symmetric connection. Similarly, if $V$ is parallel to $F$, we have

$$
\begin{aligned}
\left\{\alpha+\gamma\left(f^{4}+1\right)\right\}= & e^{q}\left[\frac{n-1}{4}\left(q^{\prime}\right)^{2}+\frac{1}{2}\left\{(n-1) \lambda_{1}+(n-2) \lambda_{2}\right\} q^{\prime}\right. \\
& \left.+\lambda_{2}^{2}+\frac{1}{2} q^{\prime \prime}+(1-n) \lambda_{1} \lambda_{2}\right]
\end{aligned}
$$


So, we also get the relation between two non-zero smooth functions $\alpha$ and $\gamma$ of the manifold $M$ with respect to a quarter-symmetric connection. Now we have a following proposition:

Proposition 1 Let $(\mathrm{M}, \mathrm{g})$ be a warped product manifold $\mathrm{B} \times_{\mathrm{f}}$ I. If the generators $\mathrm{U}, \mathrm{V}$ are parallel to $\mathrm{F}$ in a generalized quasi-Einstein manifold with respect to a quarter-symmetric connection, then we get the relation between three non-zero smooth functions $\alpha, \beta$ and $\gamma$ of the manifold $M$ with respect to a quarter-symmetric connection given by (39) and (40).

\section{Ricci-semisymmetric $\mathrm{G}(\mathrm{QE})_{\mathrm{n}}$ with respect to quar- ter symmetric connection}

A Riemannian manifold is said to be Ricci-semisymmetric if $R \cdot S=0$ holds. In this section we study Ricci-semisymmetric $G(Q E)_{n}$ with respect to quarter symmetric connection and prove the following theorem:

Theorem 3 A Ricci-semisymmetric $\mathrm{G}(\mathrm{QE})_{\mathfrak{n}}$ with respect to quarter symmetric connection is nearly quasi-Einstein manifold with respect to quarter symmetric connection under the following condition holds:

(i) $\mathrm{P} \in \chi(\mathrm{F})$ i.e., parellel vector field.

(ii) $\mathrm{f}$ is constant.

Proof. Suppose that $\breve{R} \cdot \breve{S}=0$. Then we get

$$
\breve{S}(\breve{R}(X, Y) Z, W)+\breve{S}(Z, \breve{R}(X, Y) W)=0,
$$

where $X, W \in \chi(F), Y, Z \in \chi(B)$.

From (2), we have

$$
\begin{aligned}
\breve{S}(\breve{R}(X, Y) Z, W)= & \alpha g(\breve{R}(X, Y) Z, W)+\beta g(\breve{R}(X, Y) Z, U) g(W, U) \\
& +\gamma g(\breve{R}(X, Y) Z, V) g(W, V) .
\end{aligned}
$$

Now using (35), we have

$$
\begin{aligned}
\breve{S}(\breve{R}(X, Y) Z, W)= & \alpha g(\breve{R}(X, Y) Z, W)+\beta g\left(\breve{R}(X, Y) Z, U_{B}+f^{2} U_{F}\right) g\left(W, U_{F}\right) \\
& +\gamma g\left(\breve{R}(X, Y) Z, V_{B}+f^{2} V_{F}\right) g\left(W, V_{F}\right),
\end{aligned}
$$


i. e.,

$$
\begin{aligned}
\breve{S}(\breve{R}(X, Y) Z, W)= & \alpha g(\breve{R}(X, Y) Z, W)+\beta f^{4} g\left(W, U_{F}\right) g(Y, Z)\left[\lambda_{2}^{2} \Gamma(X) g\left(P, U_{F}\right)\right. \\
& \left.-\lambda_{1} \lambda_{2} \Gamma(P) g\left(X, U_{F}\right)\right]+c f^{4} g\left(W, V_{F}\right) g(Y, Z)\left[\lambda_{2}^{2} \Gamma(X)\right. \\
& \left.g\left(P, V_{F}\right)-\lambda_{1} \lambda_{2} \Gamma(P) g\left(X, V_{F}\right)\right]
\end{aligned}
$$

Now, using the proposition 1 and proposition 3.3 in [14], we have

$$
\begin{aligned}
\breve{S}(Z, \breve{R}(X, Y) W)= & -\alpha g\left(Z,-\lambda_{1} \lambda_{2} \Gamma(P) g(X, W) Y+\lambda_{1}^{2} \Gamma(X) \Gamma(W) Y\right. \\
& +\beta g\left(Z, U_{B}\right) g\left(Y, U_{B}\right)\left[-\lambda_{1} \lambda_{2} \Gamma(P) g(X, W)\right. \\
& \left.+\lambda_{1}^{2} \Gamma(X) \Gamma(W)\right]+\gamma g\left(Z, V_{B}\right) g\left(Y, V_{B}\right) \\
& {\left[-\lambda_{1} \lambda_{2} \Gamma(P) g(X, W)+\lambda_{1}^{2} \Gamma(X) \Gamma(W)\right] . }
\end{aligned}
$$

Let $e_{i}$ be an orthonormal basis of the tangent space at each point of the manifold where $1 \leq i \leq n$. Now putting $X=W=e_{i}$ in (44) and (45) and using the equation (41) and proposition 1 and proposition 3.3 in [14], we get

$$
\begin{aligned}
\alpha \breve{S}(Y, Z) & +g(Y, Z) \beta f^{4}\left[\lambda_{2}^{2} g\left(P, U_{F}\right) g\left(P, U_{F}\right)-\lambda_{1} \lambda_{2} \Gamma(P) .1\right] \\
& +\gamma g(Y, Z) f^{4}\left[\lambda_{2}^{2} g\left(P, V_{F}\right) g\left(P, V_{F}\right)+\alpha\left[\lambda_{1} \lambda_{2} \Gamma(P) n g(Y, Z)\right.\right. \\
& \left.-\lambda_{1}^{2} \Gamma(P) g(Y, Z)\right]+\beta g\left(Z, U_{B}\right) g\left(Y, U_{B}\right)\left[\lambda_{1}^{2} \Gamma(P)\right. \\
& \left.-\lambda_{1} \lambda_{2} \Gamma(P)\right]+\gamma g\left(Z, V_{B}\right) g\left(Y, V_{B}\right)\left[\lambda_{1}^{2} \Gamma(P)\right. \\
& \left.-\lambda_{1} \lambda_{2} \Gamma(P)\right]=0
\end{aligned}
$$

i. e.,

$$
\breve{S}(Y, Z)=A^{\prime} g(Y, Z)+B^{\prime} E(Y, Z)
$$

where $A^{\prime}, B^{\prime}$ are non-zero functions and $E(Y, Z)$ is a symmetric tensor function. So, the manifold becomes nearly quasi Einstein manifold with respect to quarter symmetric connection. This completes the proof.

\section{$5 \quad \mathrm{G}(\mathrm{QE})_{\mathrm{n}}$ with the condition $\breve{\mathrm{P}} \cdot \breve{S}=0$ with respect to quarter symmetric connection}

The projective curvature tensor $\breve{\mathrm{P}}$ of type $(1,3)$ of an $n$-dimensional Riemannian manifold $\left(M^{n}, g\right),(n>3)$ with respect to quarter symmetric connection is defined by

$$
\breve{P}(X, Y) Z=\breve{R}(X, Y) Z-\frac{1}{n-1}[\breve{S}(Y, Z) X-\breve{S}(X, Z) Y]
$$


for any vector fields $X, Y, Z \in \chi(M)$.

In this section, we consider a generalized quasi-Einstein manifold satisfying the condition $\breve{P} \cdot \breve{S}=0$ with respect to quarter symmetric connection and we have a following theorem.

Theorem $4 A \mathrm{G}(\mathrm{QE})_{\mathrm{n}}$ satisfying $\breve{\mathrm{P}} \cdot \breve{\mathrm{S}}=0$ with respect to quarter symmetric connection is nearly quasi-Einstein manifold with respect to quarter symmetric connection under the following condition holds:

(i) $\mathrm{P} \in \chi(\mathrm{F})$ i.e., parallel vector field.

(ii) $\mathrm{f}$ is constant, $\mathrm{B}$ is one-dimensional base and $\mathrm{X}, \mathrm{W} \in \mathrm{X}(\mathrm{F}), \mathrm{Y}, \mathrm{Z} \in \mathrm{\chi}(\mathrm{B})$.

Proof. Suppose that

$$
\breve{\mathrm{P}} \cdot \breve{\mathrm{S}}=0 \text {. }
$$

Now using the equation (2), (44) and (35), we have

$$
\begin{aligned}
\breve{S}(\breve{P}(X, Y) Z, W)= & \alpha g(\breve{R}(X, Y) Z, W)-\frac{\alpha}{(n-1)}[\breve{S}(Y, Z) g(X, W)] \\
& +f^{2} \beta g\left(W, U_{F}\right) A(\breve{P}(X, Y) Z) \\
& +\gamma f^{2} g\left(W, V_{F}\right) B(\breve{P}(X, Y) Z),
\end{aligned}
$$

as $\breve{S}(X, Z)=0$.

Again using (48) and proposition 1 and proposition 3.3 in [14], we have

$$
\begin{aligned}
\breve{S}(\breve{P} & (X, Y) Z, W)=\alpha g(Y, Z)\left[\lambda_{1} \lambda_{2} \Gamma(P) g(X, W)-\lambda_{2}^{2} \Gamma(X) g(P, W)\right] \\
& -\frac{\alpha}{(n-1)}\left[\breve{S}_{B}(Y, Z)+\left\{(n-1) \lambda_{1} \lambda_{2}-\lambda_{2}^{2}\right\} \Gamma(P) g(Y, Z)\right] g(X, W) \\
& -\beta g\left(W, U_{F}\right) f^{4}\left[g(Y, Z)\left\{\lambda_{1} \lambda_{2} \Gamma(P) g\left(X, U_{F}\right)-\lambda_{2}^{2} \Gamma(X) g\left(P, U_{F}\right)\right\}\right. \\
& \left.+\frac{g\left(X, U_{F}\right)}{(n-1)}\left\{\breve{S}_{B}(Y, Z)+\left\{(n-1) \lambda_{1} \lambda_{2}-\lambda_{2}^{2}\right\} \Gamma(P) g(Y, Z)\right\}\right] \\
& -\gamma g\left(W, V_{F}\right) f^{4}\left[g(Y, Z)\left\{\lambda_{1} \lambda_{2} \Gamma(P) g\left(X, V_{F}\right)-\lambda_{2}^{2} \Gamma(X) g\left(P, V_{F}\right)\right\}\right. \\
& \left.+\frac{g\left(X, V_{F}\right)}{(n-1)}\left\{\breve{S}_{B}(Y, Z)+\left\{(n-1) \lambda_{1} \lambda_{2}-\lambda_{2}^{2}\right\} \Gamma(P) g(Y, Z)\right\}\right] .
\end{aligned}
$$

Similarly using the equation (2), (48), (35) and we have proposition 1 and 
proposition 3.3 in [14], we have

$$
\begin{aligned}
\breve{S}(Z, & \breve{P}(X, Y) W)=\alpha g(Y, Z)\left[\lambda_{1} \lambda_{2} \Gamma(P) g(X, W)-\lambda_{1}^{2} \Gamma(W) \Gamma(X)\right] \\
& +\frac{\alpha}{(n-1)} g(Y, Z)\left[\breve{S}_{F}(X, W)+\left\{(n-1) \lambda_{1} \lambda_{2}-\lambda_{2}^{2}\right\} \Gamma(P) g(X, W)\right] \\
& \left.+\left\{(1-n) \lambda_{1}^{2}+\lambda_{2}^{2}\right\} \Gamma(W) \Gamma(X)\right]+\beta g\left(Z, U_{B}\right) g\left(Y, U_{B}\right)\left[\lambda_{1} \lambda_{2} g(X, W) \Gamma(P)\right. \\
& \left.-\lambda_{1}^{2} \Gamma(W) \Gamma(X)\right]+\frac{\beta}{(n-1)} g\left(Z, U_{B}\right) g\left(Y, U_{B}\right)\left[\breve{S}_{F}(X, W)+\left\{(n-1) \lambda_{1} \lambda_{2}\right.\right. \\
& \left.\left.-\lambda_{2}^{2}\right\} \Gamma(P) g(X, W)+\left\{(1-n) \lambda_{1}^{2}+\lambda_{2}^{2}\right\} \Gamma(W) \Gamma(X)\right] \\
& +\gamma g\left(Z, V_{B}\right) g\left(Y, Y_{B}\right)\left[\lambda_{1} \lambda_{2} g(X, W) \Gamma(P)-\lambda_{1}^{2} \Gamma(W) \Gamma(X)\right] \\
& +\frac{\gamma}{(n-1)} g\left(Z, V_{B}\right) g\left(Y, V_{B}\right)\left[\left[\breve{S}_{F}(X, W)+\left\{(n-1) \lambda_{1} \lambda_{2}\right.\right.\right. \\
& \left.\left.-\lambda_{2}^{2}\right\} \Gamma(P) g(X, W)+\left\{(1-n) \lambda_{1}^{2}+\lambda_{2}^{2}\right\} \Gamma(W) \Gamma(X)\right] .
\end{aligned}
$$

Since, $\breve{S}(X, Y)=0$, from (51) and (52), we have

$$
\breve{S}(X, W)=A^{\prime \prime} g(X, W)+B^{\prime \prime} E(X, W),
$$

where $A^{\prime \prime}, B^{\prime \prime}$ are non-zero functions and $E(Y, Z)$ is a symmetric tensor function.

So, the manifold becomes nearly quasi-Einstein manifold with respect to quarter symmetric connection. This completes the proof.

\section{Example of warped product on generalized quasi- Einstein manifold with respect to quarter sym- metric connection}

Taking a local coordinate system in M such that $\mathrm{g}, \nabla, \breve{\nabla}, \omega, \phi, \mathrm{T}$ have the local expression, respectively, $g_{i j}, \Gamma_{j i}^{h}, \breve{\Gamma}_{j i}^{h}, \omega_{i}, \phi_{j}^{h}, T_{j i}^{h}$ then, by a direct computation, we have

$$
\mathrm{T}_{\mathrm{ji}}^{\mathrm{h}}=\omega_{j} \phi_{\mathrm{i}}^{\mathrm{h}}-\omega_{\mathrm{i}} \phi_{j}^{\mathrm{h}} .
$$

In a local coordinate, the relation between a quarter-symmetric metric connection and the Levi-Civita connection is [13],

$$
\breve{\Gamma}_{j i}^{h}=\Gamma_{j i}^{h}+\frac{1}{2} \omega_{j}\left(\phi_{k i}+\phi_{i k}\right) g^{k h}-\frac{1}{2} \omega_{i}\left(\phi_{k j}+\phi_{j k}\right) g^{k h}-\frac{1}{2} \omega_{k}\left(\phi_{j i}+\phi_{i j}\right) g^{k h}
$$


Now, we define a Riemannian metric $\mathrm{g}$ on $\mathrm{M}^{4}$ by the formula

$$
d s^{2}=g_{i j} d x^{i} d x^{j}=(1+2 p)\left[\left(d x^{1}\right)^{2}+\left(d x^{2}\right)^{2}+\left(d x^{3}\right)^{2}+\left(d x^{4}\right)^{2}\right],
$$

where $i, j=1,2,3,4$ and $x^{1}, x^{2}, x^{3}, x^{4}$ are the standard coordinates of $R^{4}$ and $p=\frac{e^{x^{1}}}{k^{2}}$ and $k$ is a non-zero constant. Then the only non-vanishing components of the Christoffel symbols, the curvature tensor, the Ricci tensor and scalar curvature are given by

$$
\begin{aligned}
& \Gamma_{22}^{1}=-\frac{p}{1+2 p}=\Gamma_{33}^{1}=\Gamma_{44}^{1}=-\Gamma_{11}^{1}=-\Gamma_{12}^{2}=-\Gamma_{13}^{3}=-\Gamma_{14}^{4}, \\
& \mathrm{R}_{1221}=R_{1331}=R_{1441}=\frac{p}{1+2 p}, \quad S_{11}=\frac{3 p}{(1+2 p)^{2}}, \\
& S_{22}=S_{33}=S_{44}=\frac{p}{(1+2 p)^{2}}, \quad r=\frac{6 p}{(1+3 p)^{3}} \neq 0 .
\end{aligned}
$$

Let us consider the 1-form and the associated tensor $\phi$ as follows:

$$
\omega_{1}=c_{1}, \omega_{2}=0, \omega_{3}=0, \omega_{4}=0,
$$

where $c_{1}$ is arbitrary scalar and

$$
\phi=\left(\phi_{i j}\right)\left(\begin{array}{cccc}
0 & \phi_{12} & \phi_{13} & \phi_{14} \\
-\phi_{12} & 0 & \phi_{23} & \phi_{24} \\
-\phi_{13} & -\phi_{23} & 0 & \phi_{34} \\
-\phi_{14} & -\phi_{24} & -\phi_{34} & 0,
\end{array}\right)
$$

where $\phi_{i j} \neq 0$, where $i, j \in\{1,2,3,4\}$, and $i \neq j$.

From (54), we have $\breve{\Gamma}_{j i}^{h}=\Gamma_{j i}^{h}$.

The non-vanishing curvature tensors and the Ricci tensors with respect to a quarter symmetric metric connection are

$$
\breve{R}_{1221}=R_{1221}, \breve{R}_{1331}=R_{1331}, \breve{R}_{1441}=R_{1441}=\frac{p}{1+2 p}
$$

and

$$
\breve{S}_{11}=S_{11}=\frac{3 p}{(1+2 p)^{2}}, \breve{S}_{22}=\breve{S}_{33}=\breve{S}_{44}=\frac{p}{(1+2 p)^{2}} .
$$

Let us now consider the associated scalars as follows:

$$
\alpha=\frac{p}{(1+2 p)^{3}}, \beta=-3, \gamma=5 p .
$$


In terms of local coordinate system, let us consider the 1-forms $A$ and $B$ as follows:

$$
A_{i}(x)= \begin{cases}\frac{\sqrt{p}}{1+2 p}, & \text { if } i=1 \\ 0, & \text { otherwise }\end{cases}
$$

and

$$
B_{i}(x)= \begin{cases}\frac{1}{1+2 p}, & \text { if } i=1 \\ 0, & \text { otherwise }\end{cases}
$$

then we have

$$
\begin{aligned}
& \breve{S}_{11}=\alpha g_{11}+\beta A_{1} A_{1}+\gamma B_{1} B_{1}, \\
& \breve{S}_{22}=\alpha g_{22}+\beta A_{2} A_{2}+\gamma B_{2} B_{2}, \\
& \breve{S}_{33}=\alpha g_{33}+\beta A_{3} A_{3}+\gamma B_{3} B_{3}, \\
& \breve{S}_{44}=\alpha g_{44}+\beta A_{4} A_{4}+\gamma B_{4} B_{4} .
\end{aligned}
$$

Since all the cases other than (i)-(iv) are trivial, we can say that

$S_{i j}=\alpha g_{i j}+\beta A_{i} A_{j}+\gamma B_{i} B_{j}$, for $i, j=1,2,3,4$.

Example 1 Let $\left(\mathrm{M}^{4}, \mathrm{~g}\right)$ be a Riemannian manifold endowed with the metric given by

$$
d s^{2}=g_{i j} d x^{i} d x^{j}=(1+2 p)\left[\left(d x^{1}\right)^{2}+\left(d x^{2}\right)^{2}+\left(d x^{3}\right)^{2}+\left(d x^{4}\right)^{2}\right],
$$

where $i, j=1,2,3,4$ and $x^{1}, x^{2}, x^{3}, x^{4}$ are the standard coordinates of $R^{4}$ and $\mathrm{p}=\frac{\mathrm{e}^{\mathrm{x}^{1}}}{\mathrm{k}^{2}}$ and $\mathrm{k}$ is a non-zero constant. Then $\left(\mathrm{M}^{4}, \mathrm{~g}\right)$ is an $\mathrm{G}(\mathrm{QE})_{4}$ with respect to quarter symmetric connection and also with nonvanishing and nonconstant scalar curvature.

So, $\left(M^{4}, g\right)$ be a Riemannian manifold endowed with the metric given by

$$
d s^{2}=g_{i j} d x^{i} d x^{j}=(1+2 p)\left[\left(d x^{1}\right)^{2}+\left(d x^{2}\right)^{2}+\left(d x^{3}\right)^{2}+\left(d x^{4}\right)^{2}\right],
$$

where $(i, j=1,2,3,4), p=\frac{e^{x^{1}}}{k^{2}}, k$ constant is $G(Q E)_{4}$ with respect to quarter symmetric connection.

Now, to define warped product on $\mathrm{G}(\mathrm{QE})_{4}$, we consider the warping function $f: R^{3} \longrightarrow(0, \infty)$ by $f\left(x^{1}, x^{2}, x^{3}\right)=\sqrt{ }(1+2 p)$ and we observe that $f>0$ is 
a smooth function. The line element defined on $R^{3} \times R$ which is of the form $B \times{ }_{f} F$, where $B=R^{3}$ is the base and $F=R$ is the fibre.

Therefore the metric $d s_{M}^{2}$ can be expressed as $d s_{B}^{2}+f^{2} d s_{F}^{2}$ i.e.,

$$
d s^{2}=g_{i j} d x^{i} d x^{j}=(1+2 p)\left[\left(d x^{1}\right)^{2}+\left(d x^{2}\right)^{2}+\left(d x^{3}\right)^{2}\right]+[\sqrt{ }(1+2 p)]^{2}\left(d x^{4}\right)^{2},
$$

which is the example of warped product on generalized quasi-Einstein manifold with respect to quarter symmetric connection.

\section{References}

[1] R. L. Bishop, B. O'Neill, Manifolds of negative curvature, Trans. Amer. Math. Soc., 145 (1969), 1-49. MR 40:4891.

[2] M. C. Chaki, R.K. Maity, On quasi-Einstein manifolds, Publications Mathematicae Debrecen, 57 (2000), 297-306.

[3] B. Y. Chen, F. Dillen, L. Verstraelen, L. Vrancken, Characterizations of Riemannian space forms, Einstein spaces and conformally at spaces, Proc. Amer. Math. Soc., 128 (2000), 589-598.

[4] U. C. De, G. C. Ghosh, Some global properties of generalized quasi Einstein manifold, Ganita, 56 (2005), 65-70.

[5] U. C. De, G. C. Ghosh, On generalized quasi Einstein manifolds, Kyungpook Math. J., 44 (2004), 607-615.

[6] M. Glogowska, On quasi-Einstein certan type hypersurfaces, Journal of Geometry and Physics, 58 (2008), 599-614.

[7] A. Gebarowski, On Einstein warped products, Tensor (N.S.), 52 (1993), 204-207.

[8] S. Golab, On semi-symmetric and quarter-symmetric linear connections, Tensor N. S., 29 (1975), 249-254

[9] B. O'Neill, Semi-Riemannian Geometry- with applications to Relativity, Pure and Applied Mathematics, 103, Academic Press, Inc., New York, 1983.

[10] M. Obata, Certain conditions for a Riemannian manifold to be isometric with a sphere, J. Math. Soc. Japan, 14 (1962), 333-340. 
[11] I. M. Singer, J.A. Thorpe, The curvature of 4-dimensional Einstein spaces, Global Analysis, Princeton University Press., (1969), 355-365.

[12] A. A. Shaikh, S.K. Hui, On some classes of generalized quasi Einstein manifolds, Commun. Korean Math. Soc., 24 (2009), 415-424.

[13] Y. Han, H. T. Yun, P. Zhao, Some invariants of quarter-symmetric metric connections under the projective transformation, Filomat, 27 (4) (2013) 679-691.

[14] Q. Qu, Y. Wang, Multiply warped products with a quarter-symmetric connection, J. Math. Anal. Appl., 431 (2015), 955-987.

[15] A. Friedmann, J. A. Schouten, Über die Geometrie der halbsymmetrischen Ubertragungen, Math. Z., 21 (1924), 211-223.

[16] S. Pahan, B. Pal, A. Bhattacharyya, Multiply warped products as quasiEinstein manifolds with a quarter-symmetric connection, Rend. Istit. Mat. Univ. Trieste, 48 (2016), 587-605.

[17] S. Pahan, B. Pal, A. Bhattacharyya, On Einstein warped products with a quarter-symmetric connection, Int. J. Geom. Methods Mod. Phys., 14 (4), 2017.

[18] S. Dey, B. Pal, A. Bhattacharyya, Warped products and quasi-Einstein metrics, Caspian Journal of Mathematical Sciences, has benn accepected for publication.

[19] M. M. Tripathi, A new connection in a Riemannian manifold, International Electronic Journal of Geometry, 1 (2008), 15-24. 